\title{
Atrial natriuretic factor and postnatal diuresis in respiratory distress syndrome
}

\author{
Henry J Rozycki, Stephen Baumgart
}

\begin{abstract}
To find out if atrial natriuretic factor plays a part in the control of urine output during the initiation alone or throughout postnatal diuresis in neonates with respiratory distress syndrome, atrial natriuretic factor concentrations and clinical and renal variables were measured prospectively three times during the first three days of life in 13 premature infants. Atrial natriuretic factor concentrations rose significantly between the first and second sample times as did the urine output and output:input ratio. By the time that the third sample was taken, atrial natriuretic factor concentration had decreased significantly since the second sample had been taken, while urine flow was maintained. All subjects initiated a spontaneous diuresis that was related to the second concentration of atrial natriuretic factor. With partial correlation analysis a significant relationship was shown between the concentration of atrial natriuretic factor and the maintenance of urine output throughout the study period. Individual hormone concentrations did not, however, correlate with simultaneous renal variables. Changes in the concentrations of atrial natriuretic factor coincided with initiation of spontaneous diuresis in babies with respiratory distress syndrome, and may have a role in the complex mechanisms that maintain this diuresis.
\end{abstract}

Newborn infants with respiratory distress syndrome have a spontaneous diuresis during the first few days of life that results in a negative balance for sodium and water. ${ }^{1}$ This diuresis may be an essential precursor of the improvement in lung function that occurs during the recovery phase of respiratory distress syndrome, ${ }^{2}$ and failure to develop an early spontaneous diuresis has been implicated in the pathogenesis of bronchopulmonary dysplasia. ${ }^{3}$ Though these observations have been confirmed in several reports, the mechanisms controlling the transient but pronounced increase in urine volume that occurs during the diuresis have not been identified.

Recently, atrial natriuretic factor has been described as a potent hormone exerting its principal effects on renal function. ${ }^{4}$ A number of these effects resemble the changes in renal function and fluid balance described in neonates during the first few days of life. Specifically, atrial natriuretic factor is released from granules in the atrial myocytes in response to a rise in atrial volume. ${ }^{5}$ This may occur in newborn infants because of a rise in atrial pressure caused by the changes in intracardiac and extracardiac blood flow after birth, or because there is a rise in atrial volume caused by a shift of intracellular fluid volume to the extracellular space during the postnatal period. ${ }^{6}$ In addition, atrial natriuretic factor induces vasodilation and increases renal blood flow in previously constricted vascular beds ${ }^{7}$; similar haemodynamic changes in the renal vasculature occur during the transition from fetal to extrauterine life. ${ }^{8}$ Infusions of atrial natriuretic factor cause pronounced natriuresis, kaliuresis, and diuresis in animals and $\operatorname{man}^{9-11}$; recent studies confirm that sodium, potassium, and water balances are negative during the spontaneous postnatal diuresis in infants. ${ }^{112}$ Finally, high concentrations of plasma atrial natriuretic factor are found in newborn infants during the first week of life. 1314

These observations have led some investigators to speculate that atrial natriuretic factor may have an important role in the initiation, ${ }^{15}$ or the subsequent maintenance, or both, of neonatal diuresis. ${ }^{16}$

This study was designed to investigate two hypotheses: firstly, that atrial natriuretic factor is associated with the initiation of the postnatal spontaneous diuresis in newborn infants; and secondly, that atrial natriuretic factor secretion maintains the diuresis until it is completed rather than acting during the initiation phase alone.

\section{Patients and methods}

To examine the hypotheses stated above, newborn infants admitted to the intensive care nurseries of the Children's Hospital of Philadelphia and the Hospital of the University of Pennsylvania were considered eligible for study if they were born weighing $1500 \mathrm{~g}$ or more (necessary to obtain the required volumes for blood samples); if they were entered into study at 12 hours of age or less; if the 5 minute Apgar score was 6 or more; if an umbilical catheter had been placed with the tip below the heart under radiographic control; if they had not been treated with diuretics or cardiotonic drugs (for example epinephrine or dopamine $)^{17}$; and if they had no congenital genitourinary or cardiac anomalies. All infants had clinical and radiological evidence of respiratory distress syndrome. All patients were nursed under radiant warmers. Medical management was conducted by house officers under the direction of the attending neonatologist. Informed consent was obtained from the parents before enrolment, 
Times samples taken

. Hours of life
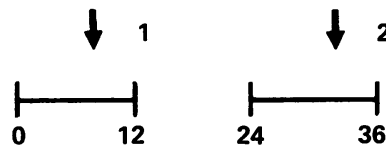

2

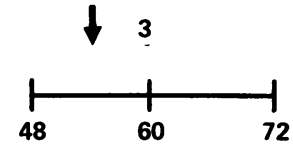

Eight hour periods

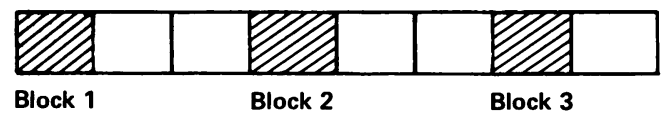

Times of serum and two hour urine collections

Delta time

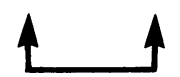

Figure 1 Diagrammatic representation of protocol. Block assignment $(1,2$, or 3$)$ of the eight hour periods depended on when the respective sample of atrial natriuretic factor was obtained. Urine and serum for chemical analyses were collected during the middle two hours of each block. Delta time was the time from the onset of diuresis (output:input $>0.8$ for a block) to the subsequent time when the next, sample of atrial natriuretic factor was taken.

and the study was approved by the Institutional Review Boards of both hospitals.

Plasma atrial natriuretic factor concentrations were determined from blood samples on three occasions: at less than 12 hours, between 24 and 36 hours, and at more than 48 hours of life. A sample of $2 \mathrm{ml}$ of blood was drawn from the umbilical catheter, put into a test tube with potassium EDTA, and immediately centrifuged at $2500 \mathrm{~g}$. After the addition of small amounts of antiproteases, the plasma was stored at $-40^{\circ} \mathrm{C}$. The samples of atrial natriuretic factor were extracted using ${ }^{18} \mathrm{C}$ Sep-pak cartridges with an $89 \%$ recovery. The extracted hormone concentrations were assayed by a specific radioimmunoassay against $\alpha$ human atrial natriuretic factor. ${ }^{5}$ The normal pool plasma atrial natriuretic factor concentration with this assay was $7 \cdot 62$ pmol/l.

Parenteral and enteral fluid intake and urine volumes were recorded hourly and cumulative totals grouped into prospective sequential eight hour periods (fig 1). Urine output was determined by weighing premeasured nappies soon after voiding. Three separate blocks were defined as the eight hour periods during which each of the three samples of atrial natriuretic factor was obtained. During the middle two hours of each block urine was collected for chemical analysis after an initial observed void into a plastic bag adherent to the perineum. The collected pooled urine and simultaneously obtained serum samples were then analysed for electrolyte and creatinine concentrations and for osmolality. Electrolyte and creatinine concentrations were measured by an Ektachem analyser (Eastman Kodak). Freezing point depression (Wescor or OsMet Precision) was used to measure osmolality.

For each block, the measured serum and urine values were used to calculate renal functions (creatinine clearance, fractional sodium excretion, and free water clearance) according to standard formulas. ${ }^{1}$ Fluid input and urine output ( $\mathrm{ml} / \mathrm{kg} /$ hour), the output:input ratio, and sodium and potassium excretion ( $\mathrm{mmol} / \mathrm{kg}$ ) hour) were also calculated.

Ventilator settings and arterial blood gases from the midpoint of each block were recorded and used to calculate the alveolar-arterial oxygen gradient $\left(\mathrm{A}-\mathrm{aDO}_{2}\right)$ (alveolar partial pressure of oxygen $\left(\mathrm{P}_{\mathrm{A}} \mathrm{O}_{2}\right)$ - arterial partial pressure of oxygen $\left(\mathrm{P}_{\mathrm{a}} \mathrm{O}_{2}\right)$; where $\mathrm{P}_{\mathrm{A}} \mathrm{O}_{2}=$ (fractional inspired oxygen $\left.\left(\mathrm{F}_{\mathrm{I}} \mathrm{O}_{2}\right) \times 713\right)-$ arterial carbon dioxide tension $\left(\mathrm{P}_{\mathrm{a}} \mathrm{CO}_{2}\right) \times\left(\mathrm{F}_{\mathrm{I}} \mathrm{O}_{2}+\right.$ $\left.\left(1-\mathrm{F}_{\mathrm{I}} \mathrm{O}_{2}\right) / 0 \cdot 8\right)$ ).

Data were analysed using the Statgraphics software (Statistical Graphics Corporation). Concentrations of atrial natriuretic factor were analysed by the Friedman test and a nonparametric Tukey test. For parametric analysis hormone concentrations were transformed logarithmically to approximate a normal distribution. Mean values for each variable over the three eight hour blocks were analysed by ANOVA for repeated measures with the subject as a covariate. The $\alpha$ error for differences in means between two sampling times was determined by the Newman-Keuls test when the ANOVA showed significant differences among all three means. A probability of $<0.05$ was accepted as significant.

In testing the first hypothesis - the relationship between atrial natriuretic factor and the initiation of diuresis - the onset of diuresis was defined as the time at the beginning the block when the output:input ratio first exceeded $0 \cdot 8 .^{2}$ This level is 1.4 standard deviations (92nd centile) above the mean of the output:input ratio preceding the diuretic period as determined by Bidiwala et al using an even stricter definition of diuresis. ${ }^{12}$ The time of onset was then related to the log of the plasma atrial natriuretic factor concentration and the other measured variables from each of the three blocks by linear regression. The differences between the second atrial natriuretic factor sample time and the onset of the diuresis for each infant was defined as delta time.

To test the second hypothesis - that atrial natriuretic factor secretion is important to maintain the diuresis - a correlation matrix including all atrial natriuretic factor concentrations and the coincident measurements of blood chemistry, calculations of renal function, and respiratory variables was constructed using linear regression. A partial correlation matrix was then constructed to delineate further the maintenance of urine volume production during diuresis as a function of selected study variables held independent from the other study variables. Urine output was correlated independently with age at time of study (hours after birth), birth weight, fluid intake, log of the plasma atrial natriuretic factor concentration, renal function (creatinine clearance, free water clearance, and fractional sodium excretion), serum sodium concentration, and severity of respiratory illness $\left(\mathrm{A}-\mathrm{aDO}_{2}\right) . \mathrm{Log}$ of the plasma atrial natriuretic factor concentration was independently tested against the same variables. Partial correlations determine the Pearson moment correlation coefficient between two variables while holding other variables constant. ${ }^{18}$

\section{Results}

Eighteen infants were enrolled in the study between April 1986 and November 1987. Three 
Table 1 Mean (SEM) concentrations of atrial natriuretic factor and fluid balance from the three study blocks

\begin{tabular}{|c|c|c|c|}
\hline & $\begin{array}{l}\text { Block } 1 \\
(n=13)\end{array}$ & $\begin{array}{l}\text { Block } 2 \\
(n=13)\end{array}$ & $\begin{array}{l}\text { Block } 3 \\
(n=11)\end{array}$ \\
\hline $\begin{array}{l}\text { Atrial natriuretic factor sample time (hour of life) } \\
\text { Atrial natriuretic factor (pmol/l) } \\
\text { Log of plasma atrial natriuretic factor (log pmol/l) } \\
\text { Fluid input (m/ } / \mathrm{kg} / \mathrm{hour} \text { ) } \\
\text { Urine output (ml/kg/hour) } \\
\text { Output:input ratio }\end{array}$ & $\begin{array}{l}10.8(0.4) \\
54.3(9 \cdot 8) \\
1.652(0.078) \\
3.09(0 \cdot 15) \\
1.31(0.32) \\
0.44(0.11)\end{array}$ & $\begin{array}{l}30.0(0 \cdot 6) \\
92 \cdot 0(13 \cdot 2)^{*} \\
1.903(0 \cdot 069)^{*} \\
3 \cdot 29(0 \cdot 15) \\
3 \cdot 11(0 \cdot 50)^{*} \\
0.94(0 \cdot 14)^{*}\end{array}$ & $\begin{array}{l}54 \cdot 8(0 \cdot 8) \\
60 \cdot 7(13 \cdot 8) \dagger \\
1.690(0 \cdot 096) \dagger \\
3.92(0 \cdot 28) \ddagger \uparrow \\
3.65(0.60) \| \\
0.91(0 \cdot 12) \|\end{array}$ \\
\hline
\end{tabular}

$\mathrm{p}<0.001$, block $2 v$ block $1 ; \dagger=\mathrm{p}<0.001$, block $3 v$ block $2 ; \ddagger=\mathrm{p}<0.02$, block $3 v$ block $1 ; \uparrow=\mathrm{p}<0.01$, block $3 v$ block 2 ; and $\|=p<0 \cdot 001$, block $3 v$ block 1 .

were excluded because their umbilical catheters were removed after the first blood samples were obtained. Two patients were withdrawn because they developed tension pneumothoraces during the study which may have acutely reduced blood return to the heart and affected atrial natriuretic factor concentrations. Two of the remaining 13 patients had only two samples of atrial natriuretic factor taken, but are included with appropriate corrections made in the analysis for missing data. Minor errors in the timing of urine and serum sampling also necessitated correction for missing data in the analysis.

The mean (SEM) weight of the 13 study subjects was 2030 (140) $\mathrm{g}$ and their gestational age was $33(0.5)$ weeks. The mean (SEM) 5 minute Apgar score was 8 (1). All infants were receiving supplementary oxygen at entry into the study and 12 received positive pressure ventilation for between 17 hours and 27 days. During the study no subject had clinical evidence of a patent ductus arteriosus, a factor known to affect atrial natriuretic factor concentrations. ${ }^{19}$

Table 1 shows the time at which each of the three samples of atrial natriuretic factor was taken. The atrial natriuretic factor (and the log of the plasma atrial natriuretic factor) concentrations rose sharply between block 1 and block $2(\mathrm{p}<0.001)$, reflecting a rise in atrial natriuretic factor observed in 12 of the 13 subjects. The hormone concentrations fell between block 2 and block $3(\mathrm{p}<\cdot 001)$. As seen in table 1 , the fluid intake rose over the three blocks, especially between blocks 2 and 3 . Urine output and output:input ratio also rose but there were no

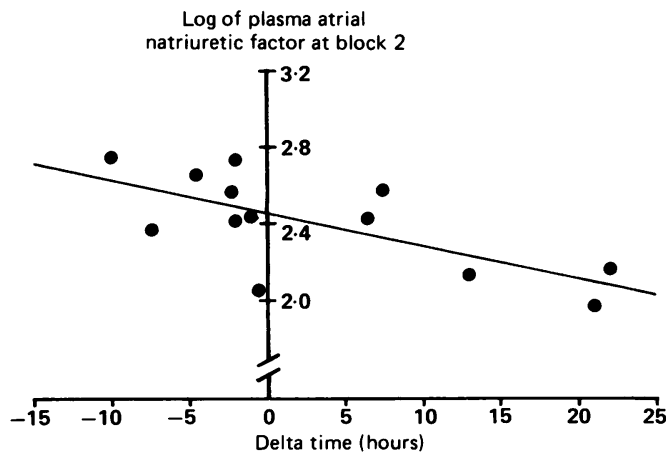

Figure 2 Log of plasma atrial natriuretic factor at block 2 plotted against the difference between the onset of diuresis and the time that the sample of atrial natriuretic factor was taken (delta time). Negative delta time indicates that the sample of atrial natriuretic factor was obtained before the onset of diuresis; positive delta time indicates that atrial natriuretic factor was sampled after onset. $Y=2 \cdot 4371-0 \cdot 1669 \mathrm{log}$ of plasma atrial natriuretic factor at block 2, $r=-0.6892$, $R^{2}=0.475, p=0.0092$. significant differences in the latter two variables between block 2 and block 3 . A continued diuresis (input:output ratio $>0.8$ ) was seen during block 3 in $7 / 11$ subjects. There were no significant differences among renal function variables (unpublished observations).

All the infants experienced a spontaneous diuresis with a mean onset of 27 (3) hours (range 7 to 43). Figure 2 shows the relationship between delta time and the log of the plasma atrial natriuretic factor concentration during block 2 . There is a significant negative correlation $(r=-0.69, p=0.0092)$ between this value and the time between the onset of diuresis and the taking of the atrial natriuretic factor sample during block 2 , indicating that atrial natriuretic factor concentrations before the onset of the diuresis were higher than those obtained after the onset.

The onset of diuresis correlated significantly with block $2 \mathrm{~F}_{\mathrm{I}} \mathrm{O}_{2}(\mathrm{r}=0.63)$, peak inspiratory pressure $(r=0.58)$, and $\mathrm{A}-\mathrm{aDO} \mathrm{O}_{2}(\mathrm{r}=0.63)$, suggesting that more severe lung disease correlated with a later onset of diuresis. There was no significant correlation between the onset of diuresis and any measured or calculated renal function or variable.

To examine the second hypothesis-that atrial natriuretic factor was associated with maintaining the diuresis until completion-the 37 atrial natriuretic factor measurements from the 13 subjects were correlated with renal and non-renal variables from the coincident block. No significant correlations were found, either by single or multiple regression. Because atrial natriuretic factor may act through a secondary messenger, cyclic guanosine monophosphate, ${ }^{20}$ and so have a delayed effect, the same variables measured eight hours later were correlated with the log of the plasma atrial natriuretic factor concentration. Again, no significant relationships were found.

Partial correlation analysis (table 2) showed that there was a significant relationship between urine output and the $\log$ of the plasma atrial natriuretic factor $(r=-0.44, p<0.01)$. Urine

Table 2 Relationship between urine output and selected variables by partial correlation analysis

\begin{tabular}{lcc}
\hline & $\begin{array}{l}\text { Correlation } \\
\text { coefficient }(r)\end{array}$ & $p$ Value \\
\hline Birth weight & -0.2733 & 0.1017 \\
Time after birth & 0.2814 & 0.0916 \\
Fluid intake volume & 0.1978 & 0.2406 \\
Log of plasma atrial natriuretic & & \\
factor & -0.4412 & $<0.01$ \\
Creatinine clearance & -0.5002 & $<0.02$ \\
Free water clearance & 0.8205 & $<0.0001$ \\
Fractional sodium excretion & 0.5243 & $<0.01$ \\
Serum sodium concentration & -0.6756 & $<0.0001$ \\
A-aDO & -0.6581 & $<0.0001$ \\
\hline
\end{tabular}


volume output was also independently related to renal functions: creatinine clearance $(r=$ $-0.50)$, free water clearance $(r=0.82)$, and fractional sodium excretion $(r=0.52)$. In addition, serum sodium concentration and alveolar arterial oxygen differences were related to urine volume. There were also significant partial correlations between the $\log$ of the plasma atrial natriuretic factor concentration and $\mathrm{A}-\mathrm{aDO}_{2}$ $(r=-0.63)$, serum sodium $(r=-0.53)$, and free water clearance $(r=0.51)$ (not listed in table 2$)$.

\section{Discussion}

The 13 infants in this investigation began their diuresis half way through the 72 hour study period. The onset of the diuresis coincided with a surge and then fall in the plasma atrial natriuretic factor concentration. In addition, infants who had blood taken for measurement of atrial natriuretic factor concentration before the onset of the diuresis had higher concentrations than those from whom samples were taken at or after the onset of diuresis. Though the results of the present study were consistent with the first hypothesis - that atrial natriuretic factor participates in initiating diuresis in infants with respiratory distress syndrome, they do not suggest that this hormone is a critical factor. If atrial natriuretic factor concentrations were low in infants who failed to achieve an early diuresis, then a more causal relationship could be implied between atrial natriuretic factor and the initiation of diuresis. The current findings may also be consistent with an increase in atrial volume initiating atrial natriuretic factor secretion and diuresis. If this latter interpretation is correct, then the continued diuresis and consequent extracellular volume contraction seen throughout block 3 should have led to a further decline in the atrial natriuretic factor concentration, as was seen in these 13 subjects.

In examining the role of atrial natriuretic factor during the maintenance of diuresis, we could find no correlation between atrial natriuretic factor concentration and any measurement of serum or urine chemistry or calculated renal function during the first 60 hours of life by regression analysis. This lack of correlation may have been the result of using differences in nappy weights and two hour urine collections to estimate renal function, which may not give a true reflection of the underlying renal function. Previous reports, however, have also found no direct and simple relationship between atrial natriuretic factor concentrations and the maintenance of diuresis or renal function. ${ }^{14} 16$

Other studies of the physiology of spontaneous diuresis in newborn infants have found that acute changes in body water distribution, ${ }^{6}$ changes in prostaglandin metabolism, ${ }^{21}$ changes in glomerular filtration and sodium excretion, 12 and changes in free water clearance, ${ }^{1}$ may influence the postnatal diuresis. These were studies of nine to 17 patients each, which were sufficient to isolate the single relationships outlined. Delineating a causal relationship between observed atrial natriuretic factor and urine volume would require over 900 babies to show a significant correlation among these variables, most probably because of the confounding effect of other factors affecting both urine volume and atrial natriuretic factor concentration. Alternatively, partial correlations can be made with a smaller number of subjects.

In the present study partial correlation analysis indicated that there was a significant relationship between urine volume and atrial natriuretic factor concentration during the study period. Significant relationships were found between urine output and creatinine clearance and serum sodium, and we also found that urine output was independent of fluid intake. These findings are consistent with a recent report by Bidiwala et al. ${ }^{12}$ In addition, this analysis highlighted the reported relationship between urine output and fractional sodium excretion and free water clearance during diuresis, which suggests an underlying change in tubular function. ${ }^{1}$

There was also a significant relationship between the atrial natriuretic factor and A$\mathrm{aDO}_{2}$ gradients as well as atrial natriuretic factor and serum sodium. The former is consistent with the findings of Shaffer et al, who noted higher atrial natriuretic factor concentrations in infants with more severe respiratory distress syndrome. ${ }^{14}$ The latter correlation is consistent with the presumed mechanism of inhibition of atrial natriuretic factor release-that is, the intravascular water volume drops resulting in increased serum sodium concentrations and a fall in the atrial natriuretic factor concentration.

Besides influencing diuresis, there are other potential hypotheses to explain the high atrial natriuretic factor concentrations found in premature infants with respiratory distress syndrome. Hypoxia enhances release of atrial natriuretic factor from the heart, and atrial natriuretic factor may function as a pulmonary vasodilator if there is hypoxic pulmonary vasoconstriction. ${ }^{22}$ Alternatively, there may be problems with regulation of atrial natriuretic factor release or end organ effect in the newborn. Both hypoxia and volume infusion increase plasma atrial natriuretic factor concentrations in fetal animals however. ${ }^{23}{ }^{24}$ Brace and Cheung infused pharmacological amounts of atrial natriuretic factor into fetal sheep, and achieved a brisk and prompt diuresis. ${ }^{25}$ Changes in intrathoracic pressure from mechanical ventilation may also be a cause of acute rises in atrial natriuretic factor concentrations in infants with respiratory distress syndrome. Application of positive end expiratory pressure in adults, however, leads to a fall in atrial natriuretic factor concentrations. ${ }^{26}$

This study provides evidence in support of both hypotheses. There was a significant rise noted in plasma atrial natriuretic factor concentrations just before and at the onset of diuresis, followed by a fall in the hormone concentration, suggesting that atrial natriuretic factor is necessary for the initiation of postnatal diuresis. It is possible, however, that the two phenomena are coincidental. Like other authors, we found no relationship found between plasma concentrations of the hormone and a number of variables that are causes or consequences of renal function. ${ }^{14} \mathrm{~A}$ partial correlation analysis, 
however, supports the hypothesis that atrial natriuretic factor may contribute to the maintenance physiology of the complete postnatal diuresis. A much larger population needs to be studied to eliminate the effect of confounding variables and accurately determine the relationship between atrial natriuretic factor and postnatal diuresis.

This study was supported in part by grants from the US National Institute of Health (Clinical Research Center Grant No RR 00240) and Merck, Sharp and Dohme Research Laboratories West Point, Pennsylvania, USA. We thank the nursing staffs of the neonatal intensive care unit and the intensive care nursery of the Children's Hospital of Philadelphia and the Hospital of the University of Pennsylvania, respectively, for their cooperation Rosetta Chiavacci RN, and Linda Corcoran RN, for technica assistance, and John C Burnett Jr, MD, of the Mayo Clinic Rochester, Minnesota, USA, for carrying out the atria natriuretic factor assays.

1 Costarino AT, Baumgart S, Norman ME, Polin RA. Renal adaptation to extrauterine life in patients with respirator distress syndrome. Am $\mathcal{F}$ Dis Child 1985;139:1060-3

2 Langman CB, Engle WD, Baumgart S, Fox WW, Polin RA. The diuretic phase of respiratory distress syndrome and its relationship to oxygenation. F Pediatr 1981;98:462-6.

3 Spitzer AR, Fox WW, Delivoria-Papadopolous $M$. Maximum diuresis: a factor in predicting recovery from respiratory distress syndrome and the development of bronchopulmonary dysplasia. $\mathcal{F}$ Pediatr 1981;98:476-80.

4 DeBold AJ, Borenstein HB, Veress AT, Sonnenberg H. A rapid and potent natriuretic response to intravenous injection of atrial myocardial extract in rats. L ife $1981 ; 28: 89-94$.

5 Burnett JC, Jr, Kao PC, Hu DC, et al. Atrial natriuretic factor elevation in congestive heart failure in the human. Science 1986;231:1145-7.

6 Cassady G. Effect of cesarean section on neonatal body water spaces. N Engl 7 Med 1971;285:887-91.

7 Maack T, Atlas SA, Camargo MJF, Cogan MG. Renal hemodynamic and natriuretic effects of atrial natriuretic hemodynamic and natriuretic effect

8 Robillard JE, Nakamura KT, Matherne GP, Jose PA. Renal hemodynamics and functional adjustments to postnatal life. Semin Perinatol 1988;12:143-50.

9 Raine AEG, Firth JG, Ledingham JGG. Renal actions of atrial natriuretic factor. Clin Sci 1989;76:1-8.
10 Weidmann P, Saxenhofer H, Ferrier C, Shaw SG. Atrial natriuretic peptide in man. Am 7 Nephrol 1988;8:1-14.

11 Zimmerman RS, Schirger JA, Edwards BS, Schwab TR, Heublein DM, Burnett JC Jr. Cardio-renal-endocrine dynamics during stepwise infusion of physiologic and pharmacologic concentrations of atrial natriuretic factor in the macologic concentrations of a

12 Bidiwala KS, Lorenz JM, Kleinman LI. Renal function correlates of postnatal diuresis in preterm infants. Pediatrics relates of posting

13 Weil J, Bidlingmaier F, Dohlmann C, Kuhnle U, Strom T, Lang RE. Comparison of plasma atrial natriuretic peptide levels in healthy children from birth to adolescence and in children with cardiac diseases. Pediatr Res 1986;20 1328-31.

14 Shaffer SG, Geer PG, Goetz KL. Elevated atrial natriuretic factor in neonates with respiratory distress syndrome. 7 Pediatr 1986;109:1028-33.

15 Kojima T, Hirata Y, Fukuda Y, Iwase S, Koyabashi Y Plasma atrial natriuretic peptide and spontaneous diuresis in sick neonates. Arch Dis Child 1987;62:667-70.

16 Ito Y, Matsumoto T, Ohbu K, et al. Concentrations of human atrial natriuretic peptide in the cord blood and plasma of the newborn. Acta Paediatr Scand 1988;77:76-8

17 Tulassay T, Rascher W, Hajdu J, Lang RE, Toth M, Seri I Influence of dopamine on atrial natriuretic peptide level in Influence of dopamine on atrial natriuretic peptide level in

18 Zar J. Biostatistical analysis. 2nd Ed. Englewood Cliffs: Prentice-Hall, 1984:339.

19 Rascher W, Seyberth HW. Atrial natriuretic peptide and patent ductus arteriosus in preterm infants. Arch Dis Child 1987;62:1165-7.

20 Ballerman BJ, Brenner BM. Biologically active atrial peptides. F Clin Invest 1985;76:2041-8.

21 Engle WD, Arant BS Jr, Wiriyathian S, Rosenfeld CR. Diuresis and respiratory distress syndrome: physiologic mechanisms and therapeutic implications. 7 Pediat 1983;102:912-7.

22 Adnot $S$, Chabrier PE, Brun-Buisson E, Viossat I, Braquet $P$. Atrial natriuretic factor attenuates the pulmonary pressor response to hypoxia. $\mathcal{f}$ Appl Physiol 1988;65:1975-83.

23 Ross MG, Ervin MG, Lam RW, Castro L, Leake RD, Fisher DA. Plasma atrial natriuretic peptide response to volume expansion in the ovine fetus. Am $\mathcal{J}$ Obstet Gynecol 1987;157:1292-7.

24 Cheung CY, Brace RA. Fetal hypoxia elevates plasma natriuretic factor concentration. Am $\mathcal{f}$ Obstet Gynecol 1988:159:1263-8.

25 Brace RA, Cheung CY. Cardiovascular and fluid responses to atrial natriuretic factor in sheep fetus. Am $\mathcal{f}$ Physio 1987;253:R561-7.

26 Leithner C, Frass M, Pacher R, Engelbert H, Pesl H Woloszczuk W. Mechanical ventilation with positive endexpiratory pressure decreases release of alpha-atrial natriuretic peptide. Crit Care Med 1987;15:484-8. 